\title{
Serum levels of nicotinamide-adenine dinucleotide phosphate oxidase 4 are associated with non-valvular atrial fibrillation
}

\author{
TONG LIU $^{1 *}$, QINGMIAO SHAO ${ }^{1 *}$, PANAGIOTIS KORANTZOPOULOS ${ }^{2}$, \\ ENZHAO LIU ${ }^{1}$, GANG XU ${ }^{1}$ and GUANGPING LI ${ }^{1}$ \\ ${ }^{1}$ Tianjin Key Laboratory of Ionic-Molecular Function of Cardiovascular Disease, Department of Cardiology, \\ Tianjin Institute of Cardiology, Second Hospital of Tianjin Medical University, Tianjin 300211, P.R. China; \\ ${ }^{2}$ Department of Cardiology, University of Ioannina Medical School, 45110 Ioannina, Greece
}

Received May 20, 2015; Accepted July 3, 2015

DOI: $10.3892 /$ br.2015.504

\begin{abstract}
Recent evidence indicates that nicotinamide-adenine dinucleotide phosphate oxidase (NOX)-derived reactive oxygen species have a pivotal role in the development of atrial fibrillation (AF). The present study aimed to investigate the potential association between serum levels of NOX4, as well as inflammatory biomarkers and AF. In total, 108 patients with $\mathrm{AF}$ (71 with paroxysmal AF and 37 with persistent/permanent $\mathrm{AF}$ ) and 68 patients without AF, as the controls, were enrolled. The demographic, clinical, laboratory, electrocardiographic and echocardiographic characteristics were carefully recorded. Serum levels of myeloperoxidase (MPO), high-sensitivity C-reactive protein (hs-CRP) and NOX4 were assessed. Left atrial diameter (LAD), left ventricular end-diastolic diameter and P-wave dispersion were significantly increased in patients with paroxysmal AF and persistent/permanent AF compared with the controls, while NOX4 levels were significantly higher in patients with paroxysmal AF and persistent/permanent AF compared to the controls $(155.57 \pm 90$ and $155.88 \pm 64.79$ vs. $126.72 \pm 23.51 \mathrm{pg} / \mathrm{ml}$, respectively, $\mathrm{P}<0.05)$. A significant correlation between serum NOX4 levels and hs-CRP, and between NOX4 levels and MPO was also evident $(r=0.170$; $\mathrm{r}=0.227, \mathrm{P}<0.05$, respectively). Multivariate analysis demonstrated that the baseline serum NOX4 level was independently associated with paroxysmal AF [odds ratio $(\mathrm{OR})=1.014$; 95\% confidence interval $(\mathrm{CI}), 1.001-1.027 ; \mathrm{P}<0.05]$ and with persistent/permanent AF (OR=1.022; 95\% CI, 1.000-1.044; $\mathrm{P}<0.05)$. There appears to be an association between increased
\end{abstract}

Correspondence to: Professor Tong Liu, Tianjin Key Laboratory of Ionic-Molecular Function of Cardiovascular Disease, Department of Cardiology, Tianjin Institute of Cardiology, Second Hospital of Tianjin Medical University, 23 Pingjiang Road, Tianjin 300211, P.R. China

E-mail: liutongdoc@126.com

${ }^{*}$ Contributed equally

Key words: oxidative stress, inflammation, atrial fibrillation, nicotinamide-adenine dinucleotide phosphate oxidase
NOX4 levels and AF, suggesting NOX4 involvement in the pathophysiology of human AF. Further studies are required to elucidate its role in atrial remodeling and to examine its potential prognostic impact.

\section{Introduction}

Atrial fibrillation (AF) represents an evolving, global epidemic that is associated with increased morbidity and mortality $(1,2)$. Recently, there has been an increasing interest for new biomarkers that may have an impact on the management and prognosis of AF (3). Notably, accumulating evidence suggests the involvement of inflammation and oxidative stress in the development and maintenance of AF (4-6). Increased levels of serum inflammatory biomarkers have been associated with AF development and perpetuation, AF burden, and with thromboembolic events (6). Additionally, excessive production of reactive oxygen species (ROS) appears to be implicated in atrial electrical and structural remodeling. The nicotinamide-adenine dinucleotide phosphate oxidases (NOXs) represent a family of multi-subunit enzymatic complexes that have a major role in the production of ROS in the cardiovascular system. In particular, the isoforms NOX2 and NOX4 are the major NOX isoforms expressed in the heart while their activation is involved in the pathogenesis of AF (7). NOX2 was upregulated in atrial tissue of a goat rapid-pacing model or in the serum of patients with paroxysmal/persistent AF $(8,9)$. With regard to NOX4, the study by Zhang et al (10) showed that $\mathrm{H}_{2} \mathrm{O}_{2}$ production in the left atrial (LA) appendage tissue was more than doubled in AF patients compared with controls, and had an association with higher NOX4 mRNA expression. In this prospective pilot observational study, the aim was to investigate the potential association between serum NOX4 levels, as well as inflammatory biomarkers and AF.

\section{Materials and methods}

Patients. In the period between August 1, 2012 and June 30, 2013, 180 consecutive patients with AF who were admitted to the Department of Cardiology, Second Hospital of Tianjin Medical University (Tianjin, China) were screened for inclusion in the study. The final study population consisted 
of 108 consecutive AF patients. The patients were divided into 3 groups: Paroxysmal AF $(n=71)$, persistent/permanent AF $(n=37)$ and patients without AF as the controls, which were matched for gender, age and atherosclerotic risk factors $(n=68)$. The control group patients were individuals who were admitted to the department during the same period for evaluation of atypical chest pain, initial evaluation of mild hypertension (no hypertensive urgencies or emergencies) or for regular clinical examination without history of arrhythmias.

AF subtypes. An electrocardiographic documentation of AF was obligatory. The subtypes of AF were defined according to European Society of Cardiology Guidelines (11). Exclusion criteria were as follows: i) Severe congestive heart failure (New York Heart Association functional class III or IV); ii) history of coronary artery bypass grafting; iii) history of myocardial infarction; iv) significant valvular heart disease; v) hypertrophic or dilated cardiomyopathy; vi) congenital heart disease; vii) serious liver or kidney dysfunction [serum creatinine $(\mathrm{Cr})$ $>3 \mathrm{mg} / \mathrm{dl}$ ]; viii) history of acute coronary syndrome in the past 6 months; ix) stroke or transient ischemic attack within the previous 3 months; $x$ ) acute infection or inflammatory/neoplastic diseases; $x i$ ) thyroid dysfunction; and xii) current smokers or those prescribed antioxidants/anti-inflammatory drugs. The study was approved by the Ethics Committee of the Second Hospital of Tianjin Medical University and written informed consent was obtained from all the patients.

Baseline characteristics. Baseline demographic and clinical characteristics were carefully recorded. White blood cell count, red blood cell distribution width, serum $\mathrm{Cr}$, fasting blood-glucose, alanine aminotransferase and aspartate aminotransferase were measured after 12-h overnight fasting. As a measure of renal function, the baseline glomerular filtration rate was estimated (eGFR) using the abbreviated Modification of Diet in Renal Disease study equation: eGFR ( $\mathrm{ml} / \mathrm{min} / 1.73 \mathrm{~m}^{2}$ of body surface area) $=186 \mathrm{x}$ (serum $\mathrm{Cr}$ in $\mathrm{mg} / \mathrm{dl})^{-1.154} \mathrm{x}$ (age in years) $)^{-0.203}$ ( $x 0.742$ in female subjects) (12). A transthoracic echocardiographic examination was performed in all the patients using the Vivid-7 system equipped with a 2.4 MHz transducer (GE Medical Systems, Milwaukee, WI, USA). LA diameter (LAD), interventricular septal thickness, left ventricular posterior wall thickness and left ventricular end-diastolic diameter were assessed. Left ventricular ejection fraction was determined from apical 4-chamber and 2-chamber views using Simpson's biplane formula. All the echocardiographic data were analyzed by the same investigator who was blinded to the clinical status of the participants. A 12-lead electrocardiogram at a signal size of $10 \mathrm{~mm} / \mathrm{mV}$ and paper speeds of $50 \mathrm{~mm} / \mathrm{sec}$ was recorded in all the patients with paroxysmal AF and controls, when the patients were in sinus rhythm. P-wave duration was measured from the beginning (junction between the isoelectric line and the beginning of P-wave deflection) and the end of the P-wave (junction between the end of P-wave deflection and isoelectric line). P-wave dispersion (PWD) was measured by calculating the difference between the duration of the longest and the shortest $\mathrm{P}$-waves in lead II in msec by one independent investigator.

Assessment of biomarkers. After 12-h overnight fasting and supine rest for $\geq 10 \mathrm{~min}$, blood samples were collected and centrifuged at $1,000 \mathrm{x}$ g for $10 \mathrm{~min}$ to obtain the supernatant, which was stored at $-80^{\circ} \mathrm{C}$ until use for the determination of specific inflammatory and oxidative biomarkers. Serum high-sensitivity C-reactive protein (hs-CRP) was measured using a high-sensitivity immmunonephelometric assay. Serum myeloperoxidase (MPO) was detected by a colorimetry method (Nanjing Jiancheng Bioengineering Institute, Nanjing, China). Serum levels of NOX4 were determined by an ELISA method (Cusabio Biotech Co., Ltd., Wuhan, China). The minimum detectable concentration of NOX4 was ranged linear from 9.38 to $600 \mathrm{pg} / \mathrm{ml}$, and intra-assay and inter-assay variabilities were $<8$ and $<10 \%$, respectively.

Statistical analysis. Categorical variables are reported as numbers (percentages) while continuous variables are mean \pm standard deviation or median (interquartile range) values. The normality of the distribution of each variable was tested by the Kolmogorov-Smirnov test. Differences between $>2$ study groups were analyzed using the one-way analysis of variance test or the non-parametric Kruskal-Wallis test as appropriate, while differences between 2 groups were assessed by the unpaired t-test or Student-Newman-Keuls-q test for continuous variables, while the $\chi^{2}$ test was used to compare categorical variables. Bivariate analysis was performed with Pearson correlation analysis. Appropriate non-parametric tests (Mann-Whitney U test and Spearman rank correlation test) were used for all the other variables. Multivariable regression analyses were performed in order to investigate the association between NOX4 and different types of AF. $\mathrm{P}<0.05$ was considered to indicate a statistically significant difference. All the tests were two-tailed and analyses were performed using SPSS 17.0 statistical package program for Windows (SPSS, Inc., Chicago, IL, USA).

\section{Results}

Clinical characteristics. The clinical characteristics of the studied patients are presented in Table I. The prevalence of cardiovascular risk factors and the associated medications were comparable between the 3 groups. There were no significant differences in hs-CRP, MPO and eGFR between the 3 groups. However, serum NOX4 levels were significantly higher in the patients with paroxysmal AF and persistent/permanent AF compared to the controls. No significant difference in serum NOX4 levels was identified between the patients with paroxysmal AF and those with persistent/permanent AF. Furthermore, correlation analysis showed a significant correlation between serum NOX4 levels and hs-CRP, and between NOX4 and MPO levels ( $r=0.17 ; r=0.227, P<0.05$, respectively).

Multivariate analysis. The multivariate analysis showed that independent predictors of paroxysmal AF were serum NOX4, LAD and PWD (Table II). By contrast, independent predictors of persistent/permanent AF were serum NOX4 and LAD (Table III).

\section{Discussion}

In the present study, an independent association was demonstrated between serum NOX4 levels and the presence of AF. 
Table I. Baseline clinical and echocardiographic characteristics of the study population.

\begin{tabular}{|c|c|c|c|c|}
\hline Characteristics & Controls $(n=68)$ & Paroxysmal AF (n=71) & Persistent/permanent AF $(n=37)$ & P-value \\
\hline \multicolumn{5}{|l|}{ Clinical features } \\
\hline Age, years & $66.4 \pm 10.6$ & $67.9 \pm 11.5$ & $67.8 \pm 10.2$ & 0.715 \\
\hline Gender, male, no. (\%) & $28(41.2)$ & $31(43.7)$ & $20(54.1)$ & 0.432 \\
\hline Hypertension, no. (\%) & $45(66.2)$ & $45(63.4)$ & $28(75.7)$ & 0.427 \\
\hline Coronary artery disease, no. $(\%)$ & $34(50.0)$ & $34(47.9)$ & $22(59.5)$ & 0.238 \\
\hline Diabetes mellitus, no. (\%) & $9(13.2)$ & $11(15.5)$ & $10(27.0)$ & 0.180 \\
\hline History of stroke, no. (\%) & $1(1.5)$ & $10(14.1)$ & $5(13.5)$ & 0.020 \\
\hline Hypercholesterolemia, no. (\%) & $23(33.8)$ & $27(38.0)$ & $12(32.4)$ & 0.807 \\
\hline Smoking habit, no. (\%) & $4(5.9)$ & $10(14.1)$ & $5(13.5)$ & 0.248 \\
\hline \multicolumn{5}{|l|}{ Laboratory examinations } \\
\hline WBC count, $10^{9} / 1^{\mathrm{a}}$ & $6.70 \pm 1.76$ & $7.62 \pm 1.23$ & $7.07 \pm 1.86$ & 0.527 \\
\hline RDW, \% CV & $12.59 \pm 0.59$ & $12.68 \pm 0.73$ & $12.72 \pm 0.60$ & 0.537 \\
\hline $\mathrm{Cr}, \mu \mathrm{mol} / 1$ & $66.12 \pm 17.86$ & $70.33 \pm 33.40$ & $71.53 \pm 17.76$ & 0.486 \\
\hline $\mathrm{eGFR}, \mathrm{ml} / \mathrm{min} / 1.73 \mathrm{~m}^{2}$ & $96.51 \pm 23.85$ & $95.84 \pm 31.19$ & $91.63 \pm 22.95$ & 0.654 \\
\hline AST, U/1 & $16.52 \pm 17.17$ & $16.91 \pm 9.02$ & $15.69 \pm 3.93$ & 0.886 \\
\hline ALT, U/l & $18.20 \pm 15.91$ & $19.18 \pm 13.84$ & $17.18 \pm 9.31$ & 0.772 \\
\hline $\mathrm{FBG}, \mathrm{mmol} / \mathrm{l}$ & $5.89 \pm 1.63$ & $6.08 \pm 1.74$ & $6.09 \pm 1.93$ & 0.780 \\
\hline $\mathrm{NOX} 4, \mathrm{pg} / \mathrm{ml}$ & $126.72 \pm 23.51$ & $155.57 \pm 90.00$ & $155.88 \pm 64.79$ & 0.038 \\
\hline hs-CRP, mg/l & $0.78(4.88)$ & $1.20(7.68)$ & $1.36(7.62)$ & 0.551 \\
\hline MPO, U/1 & $0.149 \pm 0.029$ & $0.160 \pm 0.040$ & $0.158 \pm 0.036$ & 0.177 \\
\hline PWD, msec & $47.16 \pm 13.31$ & $58.58 \pm 17.41$ & & $<0.0001$ \\
\hline \multicolumn{5}{|l|}{ Echocardiogram parameters } \\
\hline $\mathrm{LAD}, \mathrm{mm}$ & $33.89 \pm 4.86$ & $37.38 \pm 6.30$ & $44.21 \pm 6.92$ & $<0.0001$ \\
\hline LVEDD, mm & $47.19 \pm 5.51$ & $46.90 \pm 5.20$ & $50.65 \pm 6.77$ & 0.006 \\
\hline LVPWT, mm & $9.06 \pm 3.26$ & $9.32 \pm 2.28$ & $9.29 \pm 1.48$ & 0.388 \\
\hline IVST, mm & $9.09 \pm 1.05$ & $9.59 \pm 2.36$ & $9.24 \pm 1.82$ & 0.832 \\
\hline LVEF, \% & $60.93 \pm 5.58$ & $59.67 \pm 6.48$ & $54.27 \pm 10.20$ & $<0.0001$ \\
\hline \multicolumn{5}{|l|}{ Previous medication, no. (\%) } \\
\hline Aspirin & $54(79.4)$ & $55(77.5)$ & $31(83.8)$ & 0.742 \\
\hline Statins & $55(80.9)$ & $59(83.1)$ & $29(78.4)$ & 0.833 \\
\hline ACEIs/ARBs & $21(30.9)$ & $33(46.5)$ & $16(43.2)$ & 0.152 \\
\hline$\beta$-blocker & $20(29.4)$ & $23(32.4)$ & $13(35.1)$ & 0.827 \\
\hline $\mathrm{CCB}$ & $28(41.2)$ & $31(43.7)$ & $20(54.1)$ & 0.432 \\
\hline
\end{tabular}

${ }^{\mathrm{a} D a t a}$ are expressed as mean \pm standard deviation or median (range). AF, atrial fibrillation; WBC, white blood cell; RDW, red blood cell distribution width; $\mathrm{CV}$, coefficient of variation; $\mathrm{Cr}$, serum creatinine; eGFR, estimated glomerular filtration rate; ALT, alanine aminotransferase; AST, aspartate aminotransferase; FBG, fasting blood-glucose; NOX4, nicotinamide-adenine dinucleotide phosphate oxidase 4; hs-CRP, high-sensitivity C-reactive protein; MPO, myeloperoxidase; LAD, left atrial diameter; IVST, interventricular septal thickness; LVPWT, left ventricular posterior wall thickness; LVEDD, left ventricular end-diastolic diameter; LVEF, left ventricular ejection fraction; PWD, P-wave dispersion; ACEIs, angiotensin-converting enzyme inhibitors; ARBs, angiotensin receptor blocking agents; CCBs, calcium channel blockers.

To the best of our knowledge, this is the first human study implicating this novel oxidative stress marker in AF. Of note, no significant difference in NOX4 levels was evident between paroxysmal and persistent/permanent forms. However, no firm conclusions can be made regarding its association with the AF burden.

A large body of evidence indicates that apart from the triggers, AF development and perpetuation depends on the electrophysiological and structural remodeling of the atria (13). The pathophysiology of atrial remodeling is extremely complex and the molecular pathways indicated in the initiation and perpetuation of AF show a high diversity and variability across different underlying substrates $(14,15)$. During the past few years the role of pathophysiological pathways that involve inflammatory and oxidative processes is under investigation (4,16-18). NOXs have been proposed to be critical enzymatic sources of ROS and appear to have an important role in the pathogenesis of hypertension, atherosclerosis and heart failure (19-21). Thus far, a total of 5 NOX isoforms (NOX1-5) and 2 related dual oxidase enzymes have been discovered (22). The major NOX isoforms expressed in the cardiovascular system are NOX2 and NOX4, found in 
Table II. Multivariate logistic regression analysis on predictors of paroxysmal atrial fibrillation.

\begin{tabular}{|c|c|c|c|c|c|}
\hline Characteristics & $\beta$ & SE & Wald & $\mathrm{P}$-value & OR $(95 \% \mathrm{CI})$ \\
\hline LAD & 0.106 & 0.043 & 6.003 & 0.014 & $1.111(1.021-1.209)$ \\
\hline LVEDD & -0.096 & 0.054 & 3.171 & 0.075 & $0.908(0.817-1.010)$ \\
\hline LVEF & -0.042 & 0.040 & 1.101 & 0.294 & $0.959(0.886-1.037)$ \\
\hline eGFR & 0.005 & 0.008 & 0.334 & 0.563 & $1.005(0.989-1.020)$ \\
\hline PWD & 0.046 & 0.017 & 7.561 & 0.006 & $1.047(1.013-1.082)$ \\
\hline NOX4 & 0.014 & 0.007 & 4.676 & 0.031 & $1.014(1.001-1.027)$ \\
\hline hs-CRP & -0.046 & 0.056 & 0.670 & 0.413 & $0.955(0.856-1.066)$ \\
\hline
\end{tabular}

LAD, left atrial diameter; LVEDD, left ventricular end diastolic diameter; LVEF, left ventricular ejection fraction; eGFR, estimated glomerular filtration rate; PWD, P-wave dispersion; NOX4, nicotinamide-adenine dinucleotide phosphate oxidase 4; hs-CRP, high-sensitivity C-reactive protein; OR, odds ratio; $\mathrm{CI}$, confidence interval; $\beta$, regression coefficient; $\mathrm{SE}$, standard error.

Table III. Multivariate logistic regression analysis on predictors of persisitent/permanent atrial fibrillation.

\begin{tabular}{|c|c|c|c|c|c|}
\hline Characteristics & $\beta$ & SE & Wald & P-value & OR $(95 \% \mathrm{CI})$ \\
\hline LAD & 0.323 & 0.079 & 16.512 & $<0.0001$ & $1.381(1.182-1.613)$ \\
\hline LVEDD & 0.053 & 0.071 & 0.551 & 0.458 & $1.054(0.917-1.212)$ \\
\hline LVEF & -0.072 & 0.056 & 1.662 & 0.197 & $0.930(0.833-1.038)$ \\
\hline eGFR & -0.006 & 0.016 & 0.134 & 0.714 & $0.994(0.962-1.027)$ \\
\hline NOX4 & 0.022 & 0.011 & 3.967 & 0.046 & $1.022(1.000-1.044)$ \\
\hline hs-CRP & 0.034 & 0.049 & 0.499 & 0.480 & $1.035(0.941-1.139)$ \\
\hline
\end{tabular}

LAD, left atrial diameter; LVEDD, left ventricular end-diastolic diameter; LVEF, left ventricular ejection fraction; eGFR, estimated glomerular filtration rate; NOX4, nicotinamide-adenine dinucleotide phosphate oxidase 4; hs-CRP, high-sensitivity C-reactive protein; OR, odds ratio; CI, confidence interval; $\beta$, regression coefficient; SE, standard error.

endothelial cells, vascular smooth muscle cells, cardiomyocytes and fibroblasts. In addition, recent findings indicate that NOX4 predominantly generates $\mathrm{H}_{2} \mathrm{O}_{2}$ in contrast to NOX2, which generates $\mathrm{O}_{2}$ (19-21). In particular, NOX4 has been demonstrated to participate in cell differentiation (23), fibroblast proliferation (24) and myofibroblast activation (25), while it induces apoptosis, cardiac hypertrophy and fibrosis $(20,26)$.

Previous evidence implies that NOX-derived ROS have a critical role in the development and maintenance of AF7. The study by Dudley et al (27) reported that decreased nitric oxide (NO) and increased superoxide production in the LA appendage was associated with increased NOX activity in an experimental model of atrial tachy-pacing. Notably, the NOX inhibitor apocynin reduced the superoxide production by $91 \%$. Additionally, Kim et al (28) examined the sources of superoxide production from the right atrial appendage of patients undergoing cardiac surgery and reported that NOX2 was possibly the main source of atrial superoxide production in human atrial myocytes during sinus rhythm and AF. Of note, Cangemi et al (9) demonstrated that serum levels of soluble NOX2-derived peptide were upregulated in patients with paroxysmal/persistent AF compared to those with permanent AF, as well as compared to the controls. Zhang et al (10) indicated that angiotensin II significantly upregulates NOX4 expression and $\mathrm{H}_{2} \mathrm{O}_{2}$ production in atrial HL-1 cells. Therefore,
NOX4-induced $\mathrm{H}_{2} \mathrm{O}_{2}$ production may be involved in the pathogenesis of AF.

In this context, the present study supports the role of NOX4 activation in AF, suggesting an independent association. However, the cause-effect association is not clear. Whether NOX4 inhibition modulates atrial remodeling and prevents AF promotion and perpetuation is a noteworthy area for future investigation. NOX4 was also demonstrated to correlate with MPO and hs-CRP. Thus, the possibility that the upregulation of NOX4 reflects the inflammatory status, which often complicates the clinical course of AF, cannot be excluded. In addition, serum NOX4 levels between paroxysmal and persistent/permanent AF had no significant difference. A plausible explanation could be the relatively small size of the patient groups. The increase in oxidative stress is also dependent on different sources of ROS according to the type of AF. Other sources of ROS, such as uncoupled endothelial NO synthase and mitochondrial oxidases, have been indicated in the setting of permanent $\mathrm{AF}(8)$.

Previous studies have shown that increased levels of inflammatory biomarkers, including hs-CRP and MPO, are associated with an increased risk of AF $(6,29)$. They can also predict AF recurrence following successful electrical cardioversion (5) or catheter ablation (29). However, this association was not observed in the present study and may be due to the relatively small sample size and to differences in the study population. 
Certain potential limitations should be acknowledged. First, the study population was small. Second, there are no data on the exact AF burden (number of episodes and duration). Additionally, data on the prognostic significance of NOX4 in terms of future AF burden and other future untoward events is unavailable. The intracellular levels of NOX4 in order to investigate the association with plasma levels were not assessed. Finally, the effect of agents with pleiotropic properties on this oxidative stress marker was not studied.

In conclusion, there appears to be an association between increased NOX4 levels and AF, suggesting NOX4 involvement in the pathophysiology of human AF. Further studies are required to elucidate its role in atrial remodeling and to examine its potential prognostic impact.

\section{Acknowledgements}

The present study was supported by a grant (no. 81270245 to T.L.) from the National Natural Science Foundation of China.

\section{References}

1. Ball J, Carrington MJ, McMurray JJ and Stewart S: Atrial fibrillation: Profile and burden of an evolving epidemic in the 21st century. Int J Cardiol 167: 1807-1824, 2013.

2. Lip GY, Tse HF and Lane DA: Atrial fibrillation. Lancet 379: 648-661, 2012

3. Hijazi Z, Oldgren J, Siegbahn A, Granger CB and Wallentin L: Biomarkers in atrial fibrillation: A clinical review. Eur Heart J 34: $1475-1480,2013$

4. Korantzopoulos P, Kolettis TM, Galaris D and Goudevenos JA: The role of oxidative stress in the pathogenesis and perpetuation of atrial fibrillation. Int J Cardiol 115: 135-143, 2007.

5. Liu T, Li G, Li L and Korantzopoulos P: Association between $\mathrm{C}$-reactive protein and recurrence of atrial fibrillation after successful electrical cardioversion: A meta-analysis. J Am Coll Cardiol 49: 1642-1648, 2007

6. Guo Y, Lip GY and Apostolakis S: Inflammation in atrial fibrillation. J Am Coll Cardiol 60: 2263-2270, 2012.

7. Youn JY, Zhang J, Zhang Y, Chen H, Liu D, Ping P, Weiss JN and Cai H: Oxidative stress in atrial fibrillation: An emerging role of NADPH oxidase. J Mol Cell Cardiol 62: 72-79, 2013.

8. Reilly SN, Jayaram R, Nahar K, Antoniades C, Verheule S, Channon KM, Alp NJ, Schotten U and Casadei B: Atrial sources of reactive oxygen species vary with the duration and substrate of atrial fibrillation: Implications for the antiarrhythmic effect of statins. Circulation 124: 1107-1117, 2011.

9. Cangemi R, Celestini A, Calvieri C, Carnevale R, Pastori D, Nocella C, Vicario T, Pignatelli P and Violi F: Different behaviour of NOX2 activation in patients with paroxysmal/persistent or permanent atrial fibrillation. Heart 98: 1063-1066, 2012.

10. Zhang J, Youn JY, Kim AY, Ramirez RJ, Gao L, Ngo D, Chen P, Scovotti J, Mahajan A and Cai H: Nox4-dependent hydrogen peroxide overproduction in human atrial fibrillation and hl-1 atrial cells: Relationship to hypertension. Front Physiol 3: 140, 2012.

11. Camm AJ, Kirchhof P, Lip GY, Schotten U, Savelieva I, Ernst S, Van Gelder IC, Al-Attar N, Hindricks G, Prendergast B, et al; European Heart Rhythm Association; European Association for Cardio-Thoracic Surgery; ESC Committee for Practice Guidelines: Guidelines for the management of atrial fibrillation: The task force for the management of atrial fibrillation of the european society of cardiology (ESC). Europace 12: 1360-1420, 2010.
12. Levey AS, Bosch JP, Lewis JB, Greene T, Rogers N and Roth D; Modification of Diet in Renal Disease Study Group: A more accurate method to estimate glomerular filtration rate from serum creatinine: A new prediction equation. Ann Intern Med 130: 461-470, 1999.

13. Nattel S, Burstein B and Dobrev D: Atrial remodeling and atrial fibrillation: Mechanisms and implications. Circ Arrhythm Electrophysiol 1: 62-73, 2008.

14. Schotten U, Verheule S, Kirchhof $\mathrm{P}$ and Goette A: Pathophysiological mechanisms of atrial fibrillation: A translational appraisal. Physiol Rev 91: 265-325, 2011.

15. Wakili R, Voigt N, Kääb S, Dobrev D and Nattel S: Recent advances in the molecular pathophysiology of atrial fibrillation. J Clin Invest 121: 2955-2968, 2011

16. Van Wagoner DR: Oxidative stress and inflammation in atrial fibrillation: Role in pathogenesis and potential as a therapeutic target. J Cardiovasc Pharmacol 52: 306-313, 2008.

17. Negi S, Sovari AA and Dudley SC Jr: Atrial fibrillation: The emerging role of inflammation and oxidative stress. Cardiovasc Hematol Disord Drug Targets 10: 262-268, 2010.

18. Sovari AA and Dudley SC Jr: Reactive oxygen species-targeted therapeutic interventions for atrial fibrillation. Front Physiol 3: 311,2012

19. Sirker A, Zhang M and Shah AM: NADPH oxidases in cardiovascular disease: Insights from in vivo models and clinical studies. Basic Res Cardiol 106: 735-747, 2011.

20. Kuroda J, Ago T, Matsushima S, Zhai P, Schneider MD and Sadoshima J: NADPH oxidase 4 (Nox4) is a major source of oxidative stress in the failing heart. Proc Natl Acad Sci USA 107: 15565-15570, 2010.

21. Lassègue B, San Martín A and Griendling KK: Biochemistry, physiology, and pathophysiology of NADPH oxidases in the cardiovascular system. Circ Res 110: 1364-1390, 2012.

22. Lambeth JD: NOX enzymes and the biology of reactive oxygen. Nat Rev Immunol 4: 181-189, 2004.

23. Ismail S, Sturrock A, Wu P, Cahill B, Norman K, Huecksteadt T, Sanders K, Kennedy $\mathrm{T}$ and Hoidal J: NOX4 mediates hypoxia-induced proliferation of human pulmonary artery smooth muscle cells: The role of autocrine production of transforming growth factor- $\beta 1$ and insulin-like growth factor binding protein-3. Am J Physiol Lung Cell Mol Physiol 296: L489-L499, 2009.

24. Cucoranu I, Clempus R, Dikalova A, Phelan PJ, Ariyan S, Dikalov $S$ and Sorescu D: NAD(P)H oxidase 4 mediates transforming growth factor-betal-induced differentiation of cardiac fibroblasts into myofibroblasts. Circ Res 97: 900-907, 2005.

25. Hecker L, Vittal R, Jones T, Jagirdar R, Luckhardt TR, Horowitz JC, Pennathur S, Martinez FJ and Thannickal VJ: NADPH oxidase-4 mediates myofibroblast activation and fibrogenic responses to lung injury. Nat Med 15: 1077-1081, 2009.

26. Ago T, Kuroda J, Pain J, Fu C, Li H and Sadoshima J: Upregulation of Nox4 by hypertrophic stimuli promotes apoptosis and mitochondrial dysfunction in cardiac myocytes. Circ Res 106: 1253-1264, 2010.

27. Dudley SC Jr, Hoch NE, McCann LA, Honeycutt C, Diamandopoulos L, Fukai T, Harrison DG, Dikalov SI and Langberg J: Atrial fibrillation increases production of superoxide by the left atrium and left atrial appendage: Role of the NADPH and xanthine oxidases. Circulation 112: 1266-1273, 2005.

28. Kim YM, Guzik TJ, Zhang YH, Zhang MH, Kattach H, Ratnatunga C, Pillai R, Channon KM and Casadei B: A myocardial Nox 2 containing $\mathrm{NAD}(\mathrm{P}) \mathrm{H}$ oxidase contributes to oxidative stress in human atrial fibrillation. Circ Res 97: 629-636, 2005.

29. Li SB, Yang F, Jing L, Ma J, Jia YD, Dong SY, Zheng WF and Zhao LS: Myeloperoxidase and risk of recurrence of atrial fibrillation after catheter ablation. J Investig Med 61: 722-727, 2013. 